\title{
Local observable for linear lattice imperfections in circular accelerators
}

\author{
A. Wegscheider \\ CERN, Geneva 23, Switzerland \\ and Universität Hamburg, Luruper Chaussee 149, 22761 Hamburg, Germany \\ R. Tomás \\ CERN, Geneva 23, Switzerland
}

(Received 21 January 2020; accepted 21 April 2020; published 11 May 2020)

\begin{abstract}
Finding a truly local observable for perturbations of the linear beam dynamics is a nontrivial task, contrary to the nonlinear regime, where local resonance driving terms already exist. The phase beating between two locations depends on errors outside of this region. However, phase advances between four nearby locations can be arranged in a way to cancel the contributions from errors outside of this region up to first order. The resulting local observable contains valuable information about quadrupolar lattice imperfections. This report seeks to explore this local phase beating observable and to test its usefulness for gaining insight in the linear optics imperfections of a circular accelerator.
\end{abstract}

DOI: 10.1103/PhysRevAccelBeams.23.054002

\section{INTRODUCTION}

The measurement and control of the optics of an accelerator is a critical task for machine performance. In the LHC, machine protection also imposes constraints on optics aberrations. Linear optics corrections have achieved remarkable performance in the past years [1-7], pushing the precision and accuracy of $\beta$ function and transverse coupling measurements further.

Special accelerator segments like the interaction regions of colliders need a precise control of local optics which becomes a challenging task if the optics are pushed to more extreme settings. New methods and more precise hardware are required to measure and correct machine imperfections such as quadrupole errors. In the case of a collider the exact measurement and control of the $\beta$ function at the interaction point (IP) is important for operation of the machine and to optimize luminosity [8].

In order to locate error sources we are interested in local observables, i.e., terms that only depend on lattice parameters and error sources in a localized region. Such a local observable does not exist for linear lattice imperfections. For the nonlinear ones one has been found so far $[9,10]$ :

Published by the American Physical Society under the terms of the Creative Commons Attribution 4.0 International license. Further distribution of this work must maintain attribution to the author(s) and the published article's title, journal citation, and DOI.

$$
\begin{aligned}
\chi(N)= & \frac{\hat{x}_{1}(N)}{\cos \left(\varphi_{x, 12}-\frac{\pi}{2}\right)}+\frac{\hat{x}_{3}(N)}{\cos \left(\varphi_{x, 23}-\frac{\pi}{2}\right)} \\
& +\hat{x}_{2}(N)\left[\tan \left(\varphi_{x, 12}-\frac{\pi}{2}\right)+\tan \left(\varphi_{x, 23}-\frac{\pi}{2}\right)\right] .
\end{aligned}
$$

$\chi(N)$ is built with the signal of three beam position monitors (BPMs) at positions $s_{1}, s_{2}, s_{3}$. In the equation above $\hat{x}_{i} \equiv \frac{x_{i}}{\sqrt{\beta_{i}}}, \varphi_{x, i j}$ is the horizontal betatron phase advance between the BPMs at positions $i$ and $j$ and in this work we adopt the notation

$$
\varphi_{z, a b}=\varphi_{z, b}-\varphi_{z, a}
$$

as the phase advance of plane $z$ between elements $a$ and $b$.

An extension of $\chi(N)$ into the linear regime does not seem possible since measured amplitude and phase are used in a way to remove information on the linear beam dynamics.

Certain optics parameters (e.g. the $\beta$ function or coupling) can be calculated from the phase advances between two or three BPMs [11-13] independently from BPM calibration errors [14]. The phase advance measured from a Fourier transform $[15,16]$ turn-by-turn data is independent of the amplitude of the signal and, thus, not affected by calibration errors.

The phase advance between two elements of an accelerator depends, in general, on all the elements in the ring.

Under the assumption that coupling and higher order imperfections are negligible we study the effect of quadrupolar field errors on the phase advance up to first order 
and construct an observable for linear lattice imperfections that is local. For second order considerations we find a formula for phase beating but global contributions cannot be eliminated.

The focus of this work lies on circular machines where phase advance can be measured accurately by exciting an oscillation of the beam. Excitation methods include single kicks and driven oscillation by an ac dipole [17] which generate a stable coherent motion of the beam. Conceptually the local observable described in this work applies also to linear machines but accurate measurements of phase advances remain challenging and the application of the proposed technique might not be practical. Instead model-based fitting methods [18] might be more suited to retrieve optics parameters directly.

This article is organized in the following structure:

Section II derives a local expression from the phase advance beating. This expression depends solely on the phase advances between four different BPMs. It reduces to just two BPMs if their mutual model phase advance is an exact multiple of $\pi$.

Section III examines the robustness of the local observable against noise and explores the visibility of strong error sources in the arc.

Section IV shows an example of a real LHC measurement of the local observable and the results are discussed.

\section{LOCAL OBSERVABLE}

We define the phase advance beating between element $a$ and element $b$ as

$$
\Delta \varphi_{z, a b}=\varphi_{z, a b}-\varphi_{z, a b}^{\mathrm{m}} .
$$

A superscript $(m)$ denotes model values. The effect of linear lattice imperfections on the resonance driving terms (RDTs) and their impact on the betatron phase is studied. We express the betatron motion in the language of normal form and Courant-Snyder coordinates [19].

The phase beating due to quadrupolar field errors is given by

$$
\begin{aligned}
\Delta \varphi_{i j}= & \bar{h}_{i j}-8 \sin ^{2} \varphi_{i j}^{\mathrm{m}} \mathcal{R}\left\{f_{i}\right\}-8 \sin \varphi_{i j}^{\mathrm{m}} \cos \varphi_{i j}^{\mathrm{m}} \mathcal{I}\left\{f_{i}\right\} \\
& +O\left(f^{2}\right)
\end{aligned}
$$

with

$$
\begin{gathered}
\bar{h}_{i j}=\sum_{w \in I} \beta_{w}^{\mathrm{m}} \delta K_{1, w} \sin ^{2} \varphi_{w j}^{\mathrm{m}}, \\
f_{i}=f_{2000, i}=\frac{\sum_{w}^{W} K_{1, w} \beta_{x, w} \mathrm{e}^{2 i \varphi_{x, w j}}}{8\left(1-\mathrm{e}^{4 \pi i Q_{x}}\right)} .
\end{gathered}
$$

$\bar{h}_{i j}$ only depends on quadrupole errors inside the range $[i, j]$ and is therefore a local term. The RDTs $f_{i}$ in Eq. (4), on the other hand, contain global contributions. In the Appendix A the details of the derivation of Eq. (4) are carried out.

The following subsections describe the derivation of an expression for local phase beating in two distinct cases: The first is the general case with arbitrary phase advances between the BPMs. A combination of four BPMs is necessary to calculate a pure local term. The second case considers only two BPMs with a phase advance of $\pi$.

\section{A. The general case-Phase advances different from $\boldsymbol{n} \boldsymbol{\pi}$}

Equation (4) still carries a dependence on the global error distribution in the form of the terms $f_{i}$. We can eliminate those terms by carefully summing up phase advances between different pairs of BPMs as described in Appendix B and illustrated in Fig. 1.

The resummation yields an observable

$$
\begin{aligned}
\Phi_{i j k l}^{\mathrm{meas}}= & \cot \varphi_{j l}^{\mathrm{m}} \Delta \varphi_{j l}-\cot \varphi_{j k}^{\mathrm{m}} \Delta \varphi_{j k} \\
& +\cot \varphi_{i k}^{\mathrm{m}} \Delta \varphi_{i k}-\cot \varphi_{i l}^{\mathrm{m}} \Delta \varphi_{i l}
\end{aligned}
$$

which only depends on measured phase advances between the four BPMs $i, j, k$ and $l$. Up to first order it is equal to an analytic expression

$$
\begin{aligned}
\Phi_{i j k l}^{\text {model }}= & \cot \varphi_{j l}^{\mathrm{m}}\left(\bar{h}_{i l}-\bar{h}_{i j}\right)-\cot \varphi_{j k}^{\mathrm{m}}\left(\bar{h}_{i j}-\bar{h}_{i k}\right) \\
& +\cot \varphi_{i k}^{\mathrm{m}} \bar{h}_{i k}-\cot \varphi_{i l}^{\mathrm{m}} \bar{h}_{i l}
\end{aligned}
$$

which depends only on local error sources. There are no global contributions left and the two quantities defined in Eqs. (7) and (8) are truly local up to first order.

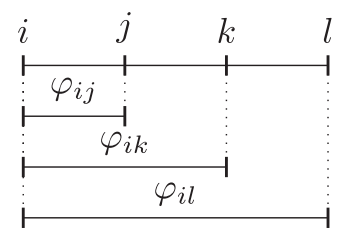

FIG. 1. The interval of BPMs with corresponding phase advances.

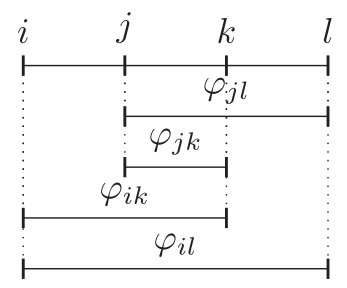

FIG. 2. The phase advances appearing in Eqs. (7) and (8). The phase advances $\varphi_{i j}$ and $\varphi_{k l}$ do not appear in the final form of the local observable. 


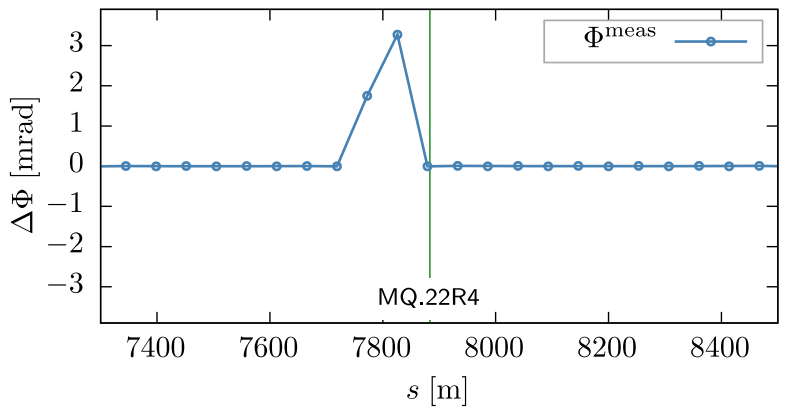

FIG. 3. The impact of a focusing error on the local observable. The plot shows an LHC arc with a relative error of $0.1 \%$ of the magnetic field of focusing quadrupole MQ.22R4 which is marked by a vertical line.

The measurement uncertainty can be propagated to $\Phi_{i j k l}^{\text {meas }}$ :

$$
\begin{aligned}
\sigma_{\Phi}^{2}= & \cot ^{2} \varphi_{j l}^{\mathrm{m}} \sigma_{\varphi_{j l}}^{2}+\cot ^{2} \varphi_{j k}^{\mathrm{m}} \sigma_{\varphi_{j k}}^{2} \\
& +\cot ^{2} \varphi_{i k}^{\mathrm{m}} \sigma_{\varphi_{i k}}^{2}+\cot ^{2} \varphi_{i l}^{\mathrm{m}} \sigma_{\varphi_{i l}}^{2} .
\end{aligned}
$$

Equation (9) is used to calculate the size of the error bars in local observable plots.

A consideration of degrees of freedom suggests that three BPMs should suffice to reconstruct the local linear optics errors. However, this reconstruction would depend on the amplitude which, in turn, may suffer from calibration errors. The local observable presented here is independent of BPM calibration errors.

Figure 3 illustrates the impact of a quadrupole error on the local observable. The plot shows an LHC arc which is constructed from 23 FODO cells of alternating focusing and defocusing quadrupoles with a total phase advance of $90^{\circ}$ and bending dipoles placed in between the quadrupoles. Two BPMs are placed in one FODO cell, directly in front of the focusing or defocusing quadrupoles, respectively. The BPMs $i, j, k$ and $l$ are chosen to be consecutive ones. A relative field error of $0.1 \%$ was introduced at magnet MQ . 22R4. Since the data points corresponding to a value of $\Phi_{i j k l}^{\text {meas }}$ are placed at the position $s_{i}$, only the three points that precede the introduced error are affected; those for which the introduced error lies in the interval $\left[s_{i}, s_{l}\right]$. The first of the three affected points has a very small value because of the proximity of the quadrupole to the BPM.

\section{B. Exact multiples of $\pi$}

If the model phase advance is $\varphi_{i j}^{\mathrm{m}}=n \pi$, the phase advance beating between two positions, Eq. (4) reduces to

$$
\Delta \varphi_{i j}=\bar{h}_{i j},
$$

which implies that $\Delta \varphi_{i j}$ is directly a local observable when $\varphi_{i j}^{\mathrm{m}}=n \pi$. In this case the number of BPMs is reduced to two at positions $i$ and $j$. In general, phase advances that are sufficiently close to multiples of $\pi$ might not be present in standard operation of an accelerator-an exception is for example the achromatic telescopic squeezing (ATS) optics [20] that is now used in LHC and which is the proposed baseline for its high luminosity upgrade-but it would be conceivable to prepare special optics settings for a corresponding measurement in any accelerator. The error of the local observable in this case,

$$
\sigma_{\Delta \varphi_{i j}}=\sigma_{\varphi_{i j}},
$$

is smaller than for the general local observable.

\section{Exploring the second order}

The details of the second order calculations can be found in Appendix C. Here we summarize the results. The detuning Hamiltonian term $h_{1100, i j}$ as well as the RDT $f_{i}$ have to be extended to second order:

$$
\begin{aligned}
h_{1100, i j} & \rightarrow h_{1100, i j}^{(1)}+h_{1100, i j}^{(2)} \\
f_{i} & \rightarrow f_{i}^{(1)}+f_{i}^{(2)} .
\end{aligned}
$$

The total phase advance beating is, then,

$$
\begin{aligned}
\Delta \varphi_{i j}= & -2 h_{1100, i j}^{(1)}-2 h_{1100, i j}^{(2)}+4 \mathcal{R}\left\{f_{j}^{(1)}-f_{i}^{(1)}\right\} \\
& +4 \mathcal{R}\left\{f_{j}^{(2)}-f_{i}^{(2)}\right\}+16\left(\mathcal{R}\left\{f_{j}^{(1)}\right\} \mathcal{I}\left\{f_{j}^{(1)}\right\}\right. \\
& \left.-\mathcal{R}\left\{f_{i}^{(1)}\right\} \mathcal{I}\left\{f_{i}^{(1)}\right\}\right)+O\left(K^{3}\right) .
\end{aligned}
$$

The same resummation techniques as for the first order will not suffice to eliminate global RDTs $f_{i}$ and $f_{j}$. If we reformulate the third line of Eq. (14) as

$$
\begin{aligned}
\mathcal{R} & \left\{f_{j}\right\} \mathcal{I}\left\{f_{j}\right\}-\mathcal{R}\left\{f_{i}\right\} \mathcal{I}\left\{f_{i}\right\} \\
& =\frac{1}{2} \mathcal{I}\left\{f_{j}^{2}\right\}-\frac{1}{2} \mathcal{I}\left\{f_{i}^{2}\right\} \\
& =\frac{1}{2} \mathcal{I}\left\{A_{i j}^{2}+2 A_{i j} f_{i} \mathrm{e}^{2 i \varphi_{i j}^{\mathrm{m}}}+\left(\mathrm{e}^{4 i \varphi_{i j}^{\mathrm{m}}}-1\right) f_{i}^{2}\right\},
\end{aligned}
$$

we see that it is not possible to separate the global $f_{i}$ from the local term $A_{i j}$. This separation was the key to be able to eliminate the global terms in the first order approximation.

For $\varphi_{i j}^{\mathrm{m}}=n \pi$ a second order term can be derived analogously:

$$
\begin{aligned}
\Delta \varphi_{i j}= & \bar{h}_{i j}-2 h_{1100, i j}^{(2)} \\
& +4 \mathcal{R}\left\{f_{j}^{(2)}-f_{i}^{(2)}\right\}+8 \mathcal{I}\left\{A_{i j}^{2}+2 A_{i j} f_{i}\right\} .
\end{aligned}
$$

This term also contains the global $f_{1}$ and $f_{2}$ and there are no common factors that can be exploited to eliminate them. 


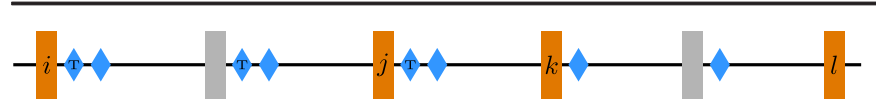

FIG. 4. The probed interval $I_{p}$ for a typical section inside an LHC arc. BPMs are represented by rectangles. The phase advance two consecutive BPMs is approximately $45^{\circ}$. Used BPMs are shown in orange, unused in gray. Blue diamonds indicate quadrupoles and trim quadrupoles. Only BPMs and quadrupoles are shown, other elements such as corrector spool pieces and the bending dipoles are omitted.

Therefore in this work purely local observable cannot be extracted from the second order phase beating.

\section{SIMULATING ERRORS AND NOISE}

\section{A. General simulation setup}

In order to assess the usability of the local observable we perform a series of simulations with different quadrupole error distributions and compare the prediction of the analytical calculations with simulated results. We base our simulations on the nominal LHC lattice at the end of run II in 2018 with ATS optics and $\beta^{*}=30 \mathrm{~cm}$.

Figure 4 shows a sketch of a typical LHC arc section. $\mathrm{BPMs}$ are placed directly in front of the quadrupoles of FODO cells. Additional trim quadrupoles (e.g. MQT) may be present.

Four cases will be studied: the first one is a set of LHC design field errors from WISE [21,22], shown in Table I, in the absence of phase noise. This setup will let us verify the equality of Eqs. (7) and (8). Then Gaussian noise of $0.7 \times 10^{-3} \times 2 \pi \mathrm{rad}$ is added to the simulated phase advances to illustrate the behavior in the presence of noise. A third simulation includes an additional strong error source in one of the quadrupoles, cf. Table II to demonstrate the impact of single strong error sources and the locality of the local observable. A last simulation setup demonstrates the visibility of quadrupolar errors originating from feed-down of sextupoles via orbit offsets.

There are many different possible combinations of BPMs. In the following comparison plots we will only show two of them: one that has a phase advance of $180^{\circ}$ in

TABLE I. Error distribution for the design LHC lattice at 6.5 TeV with weak errors in the final triplet in order to avoid higher order effects. $K$ denotes the main field component (quadrupolar field for quadrupoles, etc.).

\begin{tabular}{lc}
\hline \hline Element & $\sigma_{K_{1}} / K\left[10^{-4}\right]$ \\
\hline MQ & 12 \\
MQT & 75 \\
MQM & 12 \\
MQY & 11 \\
MQW & 15 \\
MQX & 1 \\
MB & 4 \\
\hline \hline
\end{tabular}

TABLE II. In addition to design LHC errors distribution we introduced a single strong error source in arc 45 .

\begin{tabular}{lcc}
\hline \hline Element & $\delta K_{1} / K\left[10^{-4}\right]$ & $\sigma_{K_{1}} / K\left[10^{-4}\right]$ \\
\hline MQ.22R4.B1 & 100 & $\ldots$ \\
MQ & $\ldots$ & 12 \\
MQT & $\ldots$ & 75 \\
MQM & $\ldots$ & 12 \\
MQY & $\ldots$ & 11 \\
MQW & $\ldots$ & 15 \\
MQX & $\ldots$ & 1 \\
MB & $\cdots$ & 4 \\
\hline \hline
\end{tabular}

one of the phase advances appearing in Fig. 5 and one that avoids such a term and, additionally, the 2-BPM combination with $\varphi_{i j}=180^{\circ}$.

In a real measurement we would consider only the combinations of closest BPMs to avoid the accumulation of systematic errors coming from other lattice elements and therefore we limit our study to only those combinations. The phase advance over two FODO cells in telescopic arcs of the ATS optics is tightly matched to $\pi$. On the one hand this provides a continuous set of combinations with similar phase advances and thus we can more easily compare the values of $\Phi_{i j k l}^{\text {model }}$ and $\Phi_{i j k l}^{\text {meas }}$ at different positions. On the other hand this gives rise to model phase advances close to multiples of $\pi$ for several combinations which gives the possibility to explore these cases. Table III shows the closest combinations with all occurring model phase advances. Reflected combinations are omitted. Model phase advances close to $n \pi$ cause the cotangent terms to diverge. They are therefore highlighted in red. Since we still want to study these cases and avoid divergences and the resulting numerical instabilities we impose a filter on the phase advances. Those which are closer to $n \pi$ than $10^{-6} \times 2 \pi$ are excluded. The case $45^{\circ}-90^{\circ}-45^{\circ}$ is sketched in Fig. 5.

Model and measurement values are shown for the combinations $45^{\circ}-45^{\circ}-45^{\circ}$ and $90^{\circ}-45^{\circ}-45^{\circ}$ and $\varphi_{i j}^{\mathrm{m}}=180^{\circ}$ in Figs. 6-8. Since we get the phase advances of Table III only for telescopic arcs and for the sake of readability we limit the plot region to just one telescopic arc, the one between IR4 and IR5. For simplicity we show only results for the horizontal plane.

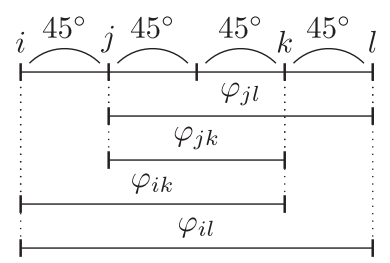

FIG. 5. The phase advances of the combination $\varphi_{i j}=45^{\circ}$, $\varphi_{j k}=90^{\circ}, \varphi_{k l}=45^{\circ}$. The phase advance $\varphi_{i l}=180^{\circ}$ causes $\cot \varphi_{j l}^{\mathrm{m}}$ to diverge. 
TABLE III. Indices $i, j, k, l$ and phases appearing model phase advances for the closest combinations. The actual model phase advances depend on the respective model settings and differ slightly from the exact values above.

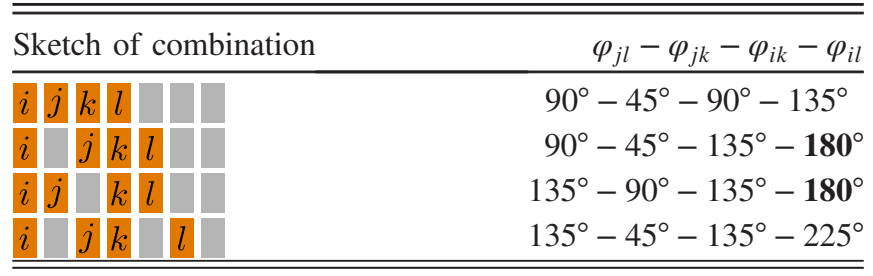

\section{B. Design field errors}

The first case, Fig. 6, is free of noise with the quad error distribution of Table I. The agreement between analytical and measurement values is excellent, showing the validity of Eqs. (7), (8) and (10).

To examine the behavior of the local observable in the vicinity of $n \pi$ we have to scan the accelerator for available model phase advances. For each BPM $i$ we are looking for a second one-BPM $j$-that is placed at $\Delta \varphi_{i j}=\pi \pm \delta \phi$ downstream. $\delta \phi$ is a threshold parameter controlling how many local observable pairs are accepted. We chose $\delta \phi=1 \times 10^{-3} \times 2 \pi$. The telescopic arcs of the ATS optics provide the needed model phase advances.

The agreement is, as for the general case, very good in the absence of errors.

\section{Phase noise}

The noise to signal ratio decreases with increasing oscillation amplitude and thus with increasing $\beta$ function at the BPM. In the LHC FODO cells BPMs are installed close to the focusing and defocusing quadrupoles and those lie at $\beta$ function maxima and minima, respectively. Therefore we can divide the arc BPMs in two categories, those with low $\beta$ function and those with high $\beta$ function. The $\beta$ function minima are usually around $30 \mathrm{~m}$ and the maxima at $180 \mathrm{~m}$. The phase advance uncertainties $\sigma_{\varphi_{i j}}$ fall into three categories: both BPMs have high $\beta$ function, only one of them has high $\beta$ and both have low $\beta$.

For this set of simulations we introduce phase noise which corresponds to noise values of the LHC signal that we typically achieve taking five data acquisitions and after cleaning [23] and harmonic analysis. We group BPMs into the before mentioned categories and apply a Gaussian error distribution to the phase values according to measurement statistics.

With the introduced phase noise the agreement decreases significantly (cf. Fig. 7). The noise is of the same order of magnitude as $\Phi_{i j k l}^{\text {meas }}$ itself. Therefore the LHC arc quadrupolar errors cannot be identified with this phase advance resolution. The combination $45^{\circ}-45^{\circ}-45^{\circ}$ shows the worst behavior under noise because the $\beta$ function alternates between high and low values from BPM to BPM and

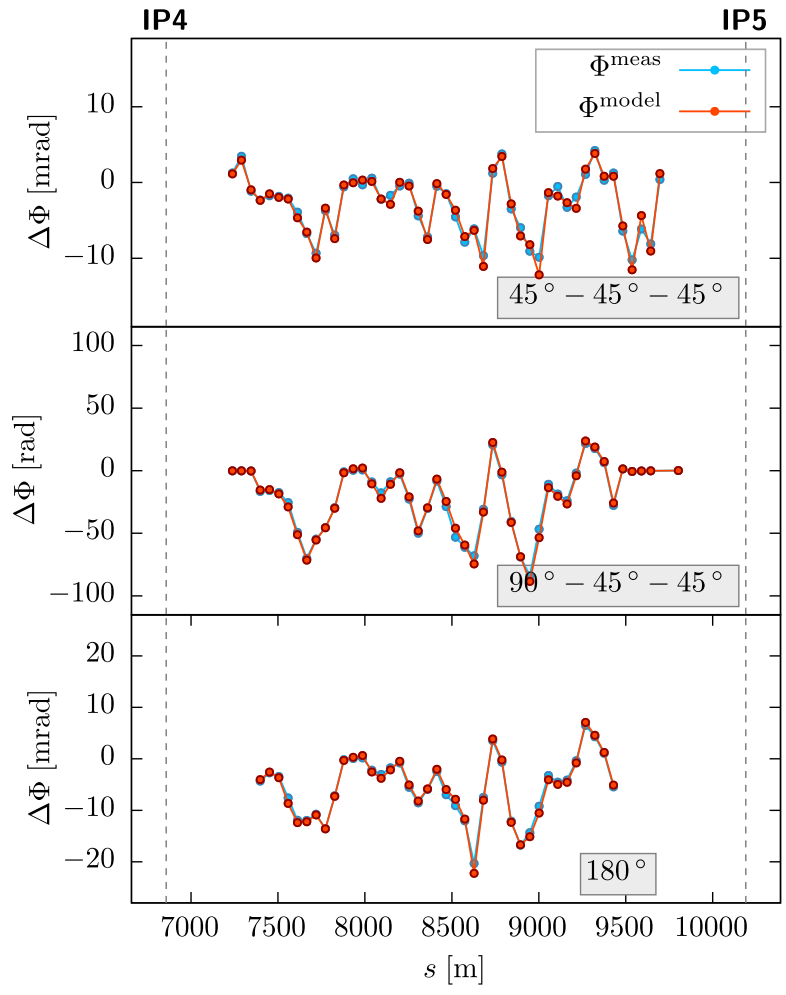

FIG. 6. This figure shows the first two combinations of Table III and the case $\varphi_{i j}^{\mathrm{m}}=\pi$ from simulations. Top: the combination $45^{\circ}-45^{\circ}-45^{\circ}$. Center: the combination $90^{\circ}-45^{\circ}-45^{\circ}$. The absolute value of the local observable in the telescopic arc (right of IP4) is 4 orders of magnitude higher than in the top plot. The plots only show values where the phase advances do not differ more than $1^{\circ}$ from the target values displayed in Table III in order to ensure comparability between the values. Additionally values with a model phase advance in $n \pi \pm 10^{-6}$ are excluded to avoid numerical instabilities. This causes the IR to be empty of local phase advances. Note that in the middle plot the values are 4 orders of magnitude higher than in the other two. This originates from the $\cot \varphi_{i l}^{\mathrm{m}}$ terms which are high because of $\varphi_{i l}^{\mathrm{m}} \approx \pi$. The bottom plot shows the local observable for model phase advances of $180^{\circ}$. In all three plots the agreement between model and simulation is excellent.

so within the four neighboring BPMs there are always two with low $\beta$ function.

The case of exact $\pi$ phase advances shows the smallest errors as only one phase advance error enters in the error propagation.

\section{Single strong error source}

For the next simulation, we assume that there is a single strong error in one of the quadrupoles. We assign $1 \%$ of relative error to MQ.22R4 to show the effect of a strong error source. Figure 8 shows that this error creates a visible peak in the local observable. The peak in the local observable is situated immediately in front of the location of the error source because the plot shows the local 


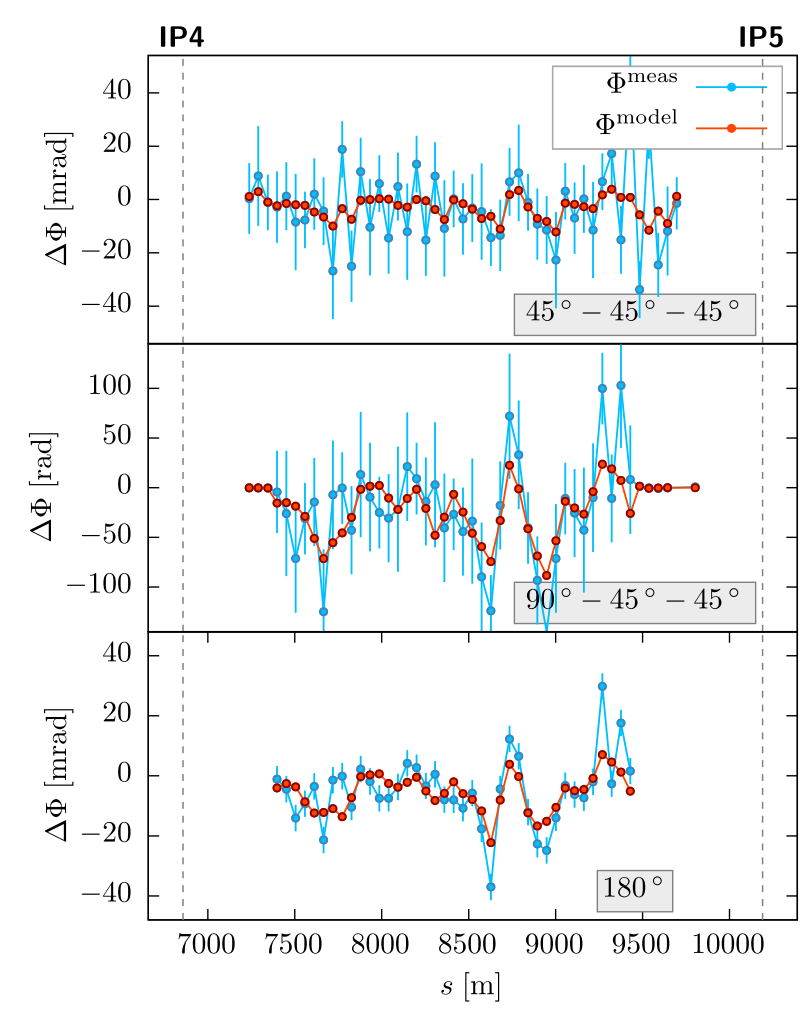

FIG. 7. Similar plots as in Fig. 6 but including a phase error of $0.7 \times 10^{-3} \times 2 \pi$ rad for high $\beta$ function values and $1.8 \times$ $10^{-3} \times 2 \pi$ rad for low $\beta$ s. The agreement between model and simulation and measurement is highly deteriorated. The error bars have been calculated using Eqs. (9) and (11). The case $\varphi_{i j}^{\mathrm{m}}=\pi$ is affected less by the error since only one phase advance error is propagated.

observable at the position of BPM $i$ but the errors of the interval $\left(s_{i}, s_{l}\right)$ enter in the calculation of the observable.

In the presence of model phase advances close to $n \pi$ the values of the local observable in the telescopic arcs are clearly enhanced (cf. bottom plot of Figs. 6 and 7).

The simulations above show that strong quadrupolar error sources $(\geq 1 \%)$ can be detected with the local observables under the studied phase resolution. For the detection of smaller errors a higher precision of the phase measurement would be needed. More precise BPMs like DOROS-BPMs [24] currently installed in the LHC interaction region and a higher excitation amplitude as well as the acquisition of a higher number of turns can increase the resolution of the phase measurement.

\section{E. Feed-down from sextupoles}

As a final test case we introduce orbit offset into the machine around the IPs by activating dedicated dispersion bumps. These are used in the ATS optics to compensate dispersion created by the crossing angles at the IPs. The transverse displacement of the beam creates quadrupolarlike errors inside sextupoles via feed-down:

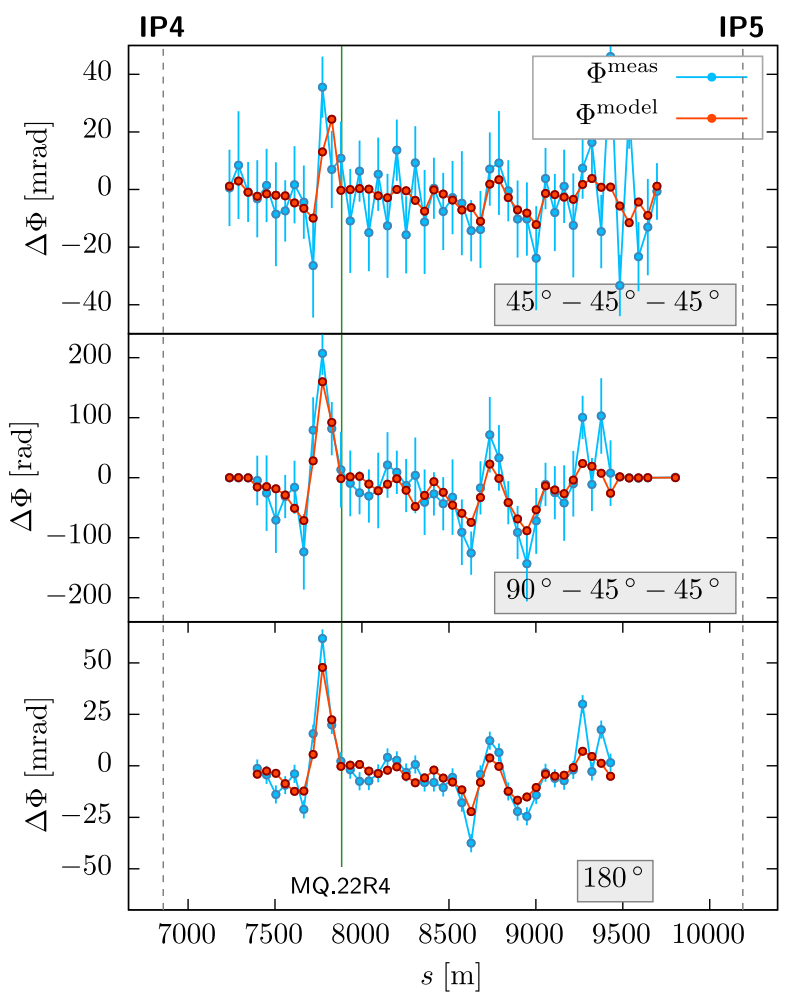

FIG. 8. The local observable with the error distribution of Table II, including a strong error source at MQ.22R4.B1 and phase noise of $0.7 \times 10^{-3} \times 2 \pi \mathrm{rad}$. The position of the strong error source is marked by a green line.

$$
\Delta K_{1, \mathrm{sext}}=\delta x K_{2}
$$

where $\delta x$ denotes horizontal offset and $K_{2}$ is the strength of the sextupole. $K_{1, \text { sext }}$ can now be used for the calculation of the local observable.

Figure 9 shows the local observable in this case. Regular bumps created by the feed-down appear which are consistent in all three cases but less pronounced in the nearest neighbors case. With the given noise level those bumps can be measured. The local observable is not affected by feeddown in the center of the arc because sextupoles at places with high orbit offset are turned off. In comparison to the previous examples, Figs. 7 and 8, the values of the local observable did not change in this region.

The bottom plot of Fig. 9 shows the changed orbit for reference. The peaks of the local observable can be identified with the peaks in the orbit. Again the local observable is in advance of the error source.

\section{EXPERIMENTAL VERIFICATION}

We calculate the local observable from a measurement taken during the LHC beam commissioning in 2018.

The measurement can be seen in Fig. 10 for the combinations $45^{\circ}-45^{\circ}-45^{\circ}$ (top plot) and $90^{\circ}-45^{\circ}-45^{\circ}$ (bottom plot). 

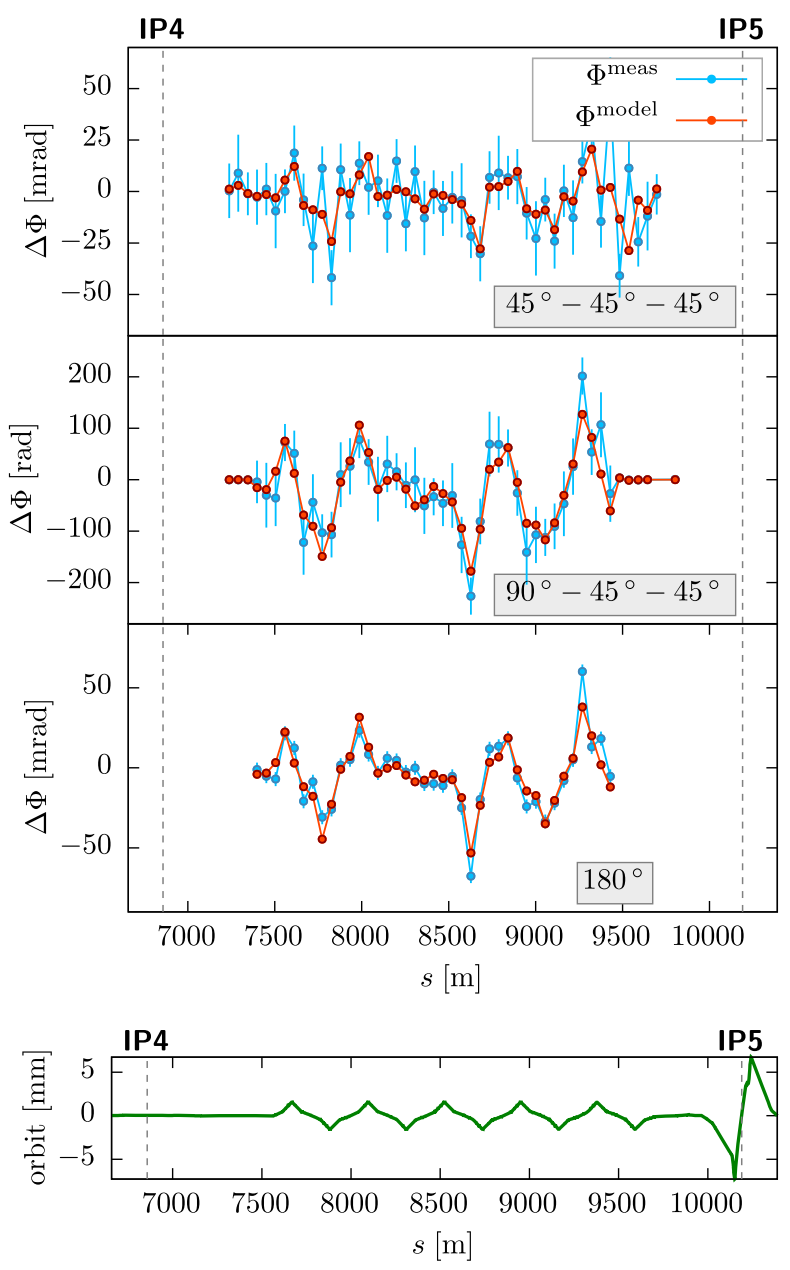

FIG. 9. The local observable with the error distribution of Table I and phase noise of $0.7 \times 10^{-3} \times 2 \pi \mathrm{rad}$. Additionally the orbit has been changed by dispersion bumps. The orbit offset creates feed-down from sextupoles.

We take a model lattice where dispersion bumps are turned off in order to see the impact on the local observable. As discussed in Sec. III, the feed-down of sextupole fields due to the orbit offset of those bumps changes the local observable. Figure 10 shows in blue the measured local observable which features similar spikes as the simulation (cf. Fig. 9). In red the effect of feed-down from sextupoles via the orbit offset on the local observable is shown. The feed-down has been calculated by introducing the dispersion bump knob into the model that was turned on during the measurement and calculating $\delta K_{1, \text { sext }}$ from Eq. (17). The pattern of the model values is also present in the measurement which confirms that their origin is indeed the feed-down. Since the $\Phi_{i j k l}^{\text {model }}$ contains only the expected feed-down from sextupoles and no other error sources (like normal quadrupole imperfections) the difference between model and measurement is then the actual local observable created by those error sources. The center region of the arc is free of a high peak because, as in the simulation, no sextupole is active at high orbit offsets.

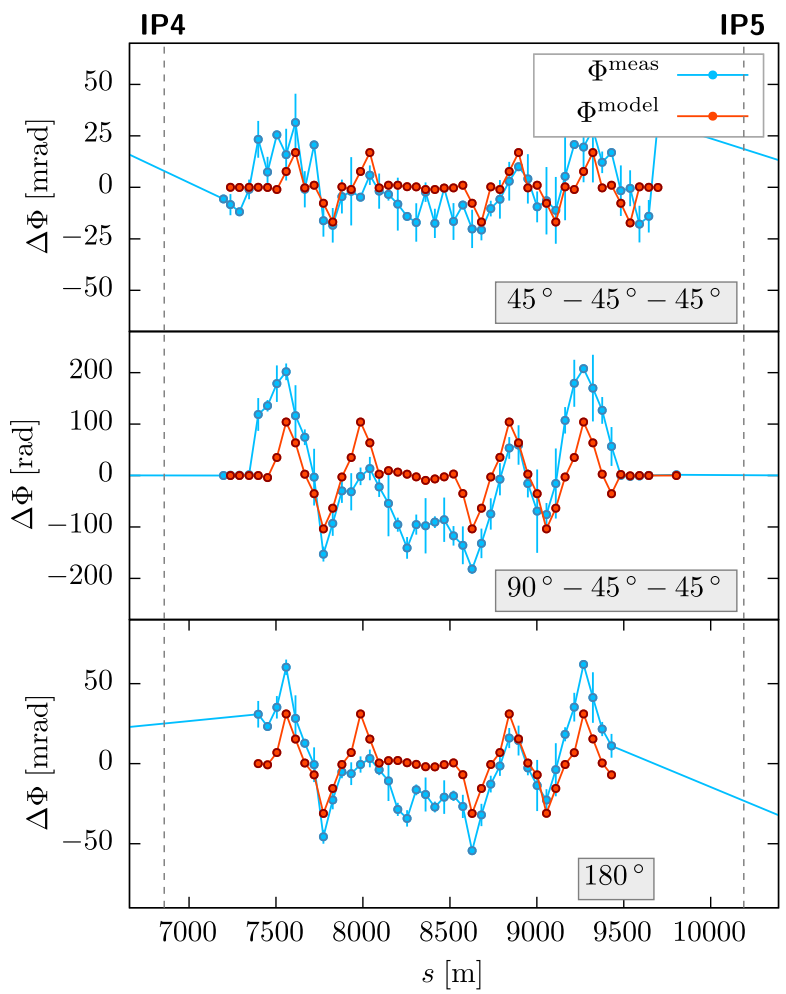

FIG. 10. Plot of measurement data of the LHC commissioning 2018 at $\beta^{*}=30 \mathrm{~cm}$. The error bars include statistical errors obtained from the phase measurement of the FFT. The effect of dispersion bumps on the local observable is clearly visible and matches well the prediction. The center region shows only a small effect from sextupoles.

\section{CONCLUSION AND OUTLOOK}

We showed the existence of a local observable for linear lattice imperfections in circular accelerators. The locality of the observable holds up to first order in the quadrupole error $\delta K_{1}$.

Phase measurement noise is an issue with the current precision of turn-by-turn measurements and a higher resolution in the measurement would be of advantage. For certain use cases, new techniques are needed to improve the control of the machine and hardware upgrades are a justified solution. Simulations show that strong error sources can be identified even with current precision of LHC measurements as they generate distinguishable peaks.

The calculated local observable of an actual measurement shows a picture that is compatible with simulations. Feed-down of orbit offsets via sextupoles can be seen in measurement data and be reproduced in simulations.

\section{ACKNOWLEDGMENTS}

We thank the LHC optics measurements and corrections team, especially T. Persson, E. Maclean and M. Hofer for fruitful discussions and A. Franchi for discussions concerning resonance driving terms. This work has been 
sponsored by the Wolfgang Gentner Programme of the German Federal Ministry of Education and Research (Grant No. 05E15CHA).

\section{APPENDIX A: INFLUENCE OF LINEAR LATTICE IMPERFECTIONS ON BETATRON PHASES}

This section revises the derivation of phase advance beating from quadrupolar field errors by reproducing the steps of $[25,26]$. We consider normal quadrupolar field errors only. In this case the generating function $F$ reads

$$
F\left(s_{j}\right)=f_{2000, j}\left(\zeta_{x, s_{j}}^{+}\right)^{2}+f_{0200, j}\left(\zeta_{x, s_{j}}^{-}\right)^{2},
$$

where the RDT $f_{j k l m}$ up to first order at the position $i$ is given by

$$
f_{j k l m, a}=\frac{-\sum_{w} h_{w, j k l m} \mathrm{e}^{i\left[(j-k) \varphi_{x, w a}+(l-m) \varphi_{y, w a}\right]}}{1-\mathrm{e}^{i\left[(j-k) Q_{x}+(l-m) Q_{y}\right]}} .
$$

For normal multipole components $K_{n}$ the coefficient $h_{w, j k l m}$ reads

$$
h_{w, j k l m}=\frac{-K_{w, n-1} i^{l+m}\left(\beta_{w, x}\right)^{\frac{j+k}{2}}\left(\beta_{w, y}\right)^{\frac{l+m}{2}}}{j ! k ! l ! m ! 2^{j+k+l+m}} .
$$

The complex Courant-Snyder coordinates can be calculated from the normal form coordinates by $[19,27]$

$$
\begin{aligned}
h_{x}^{+}\left(s_{j}, N\right)= & e^{: F: \zeta_{x, s_{j}}^{+}(N)} \\
= & \zeta_{x, s_{j}}^{+}(N)+4 i f_{2000, j}^{*} \zeta_{x, s_{j}}^{-}(N) \\
& +\left|4 f_{2000, j}\right|^{2} \zeta_{x, s_{j}}^{+}(N)+O\left(f^{3}\right),
\end{aligned}
$$

where $: F: x=[F, x]$ and the normal form coordinate in turn $N$ is $\zeta_{x, s_{j}}^{ \pm}(N)=\zeta_{x, s_{j}}^{ \pm} e^{2 \pi N Q_{x}}$. The normal form coordinates $\zeta_{z}^{ \pm}$are given by [9]

$$
\zeta_{z, s}^{ \pm}=\sqrt{2 I_{z}} \mathrm{e}^{\mp i\left(\psi_{z}+\psi_{0}\right)}, \quad z \in\{x, y\},
$$

where $s$ is the longitudinal position in the ring, $I_{z}$ is the linear invariant, and $Q_{z}$ is the tune of plane $z$.

We used the fact that $f_{2000}^{*}=f_{0200}$ to simplify the expression. The $O\left(f^{3}\right)$ term collects all third order contributions of $\mathcal{R}\left\{f_{2000, j}\right\}, \mathcal{I}\left\{f_{2000, j}\right\}$ and $\left|f_{2000, j}\right|$.

We are interested in the phase of the real signal $\hat{x}_{j}=$ $\mathcal{R}\left\{h_{x}^{+}\left(s_{j}\right)\right\}$ which reads, up to first order,

$$
\hat{x}_{j}=\mathcal{R}\left\{\left(1+4 i f_{2000, j}^{*}\right) \sqrt{2 I_{x}} \mathrm{e}^{-i\left[N Q_{x}+\psi_{x, 0 j}\right]}\right\} .
$$

The effect of the RDTs $f_{j k l m, i}$ on the phase of the main tune line is the argument of the term in parentheses in Eq. (A5):

$$
\begin{aligned}
& \arg \left(1+4 i f_{2000, j}^{*}\right) \\
& \quad=\operatorname{atan}\left(\frac{-4 \mathcal{R}\left\{f_{2000, i}\right\}}{1+4 \mathcal{I}\left\{f_{2000, j}+\left|4 f_{2000, j}\right|^{2}\right\}}\right) \\
& \quad \approx-4 \mathcal{R}\left\{f_{2000, i}\right\}-16 \mathcal{R}\left\{f_{2000, i}\right\} \mathcal{I}\left\{f_{2000, i}\right\} .
\end{aligned}
$$

Since only $f_{2000}$ appears in the phase beating we will suppress the indices 2000 from now on. A detuning $\Delta Q_{z}$ is generated by the phase independent Hamiltonian terms $h_{w, i i j j}$. The only quadrupolar contribution comes from the term $h_{1100}$. The tune in Eq. (A5) consists of

$$
Q_{x}=Q_{x}^{\mathrm{m}}+\Delta Q_{x}
$$

where $Q_{x}^{\mathrm{m}}$ is the model horizontal tune and

$$
2 \pi \Delta Q_{x}=-\frac{\partial\langle H\rangle_{\varphi}}{\partial J_{x}}=-\frac{\partial 2 J_{x} h_{1100}}{\partial J_{x}}=-2 h_{1100}+O\left(J_{x}\right) .
$$

The Hamiltonian of the whole accelerator reads

$$
\begin{aligned}
H\left(s_{a}\right)= & \sum_{w}^{W} \sum_{n} \sum_{j+k+l+m=n} h_{w, j k l m} \mathrm{e}^{i\left[(j-k) \varphi_{x, w a}+(l-m) \varphi_{y, w a}\right]} \\
& \times\left(h_{x}^{+}\right)^{j}\left(h_{x}^{-}\right)^{k}\left(h_{y}^{+}\right)^{l}\left(h_{y}^{-}\right)^{m}
\end{aligned}
$$

with $h_{z}^{ \pm}$being the normalized complex Courant-Snyder coordinate,

$$
h_{z}^{ \pm}=\hat{z} \pm i \hat{p}_{z},
$$

and $n$ denotes the order of the magnetic component ( 2 for quadrupole, 3 for sextupole, etc.). The sum over $w$ in Eqs. (A1) and (A9) runs over each element $w$ in the accelerator. The accumulated phase shift due to the detuning between elements $i$ and $j$ reads

$h_{1100, i j} \equiv-\frac{\operatorname{sgn}(j-i)}{4} \sum_{I} \beta_{w, x}^{\mathrm{m}} \delta K_{1, w}+O\left(\delta K_{1}^{2}\right)$.

$I$ is the interval $[\min (i, j), \max (i, j)]$ and $\operatorname{sgn}(x)$ denotes the sign function:

$$
\operatorname{sgn}(x)= \begin{cases}-1 & \text { if } x<0 \\ 0 & \text { if } x=0 \\ 1 & \text { if } x>0\end{cases}
$$

The total phase advance beating is then the sum of the accumulated detuning from Hamiltonian terms and the phase beating from the RDTs: 


$$
\Delta \varphi_{x, i j}=-2 h_{1100, i j}-4 \mathcal{R}\left\{f_{j}-f_{i}\right\}+O\left(f^{2}\right) .
$$

With the following identity $[9,10]$,

$$
f_{j}=\operatorname{sgn}(j-i) \frac{1}{8} \sum_{w \in I} \beta_{w}^{\mathrm{m}} \delta K_{1, w} \mathrm{e}^{2 i \varphi_{w j}^{\mathrm{m}}}+f_{i} \mathrm{e}^{2 i \varphi_{i j}^{\mathrm{m}}},
$$

we can eliminate $f_{j}$ from Eq. (A13). Here and in the following we consider only the horizontal plane and thus we omit the index $x$ in the optical functions $\phi_{a b}, \beta_{a}$ and the quadrupole field $K_{1, a}$. For compactness we rename the first part of $f_{j}$ to

$$
A_{i j}=\operatorname{sgn}(j-i) \frac{1}{8} \sum_{w \in I} \beta_{w}^{\mathrm{m}} \delta K_{1, w} \mathrm{e}^{2 i \varphi_{w j}^{\mathrm{m}}}
$$

We can simplify the last term of Eq. (A13) [25]:

$$
\begin{aligned}
\mathcal{R}\left\{f_{j}-f_{i}\right\} & =-\mathcal{R}\left\{A_{i j}\right\}+\mathcal{R}\left\{\mathrm{e}^{2 i \varphi_{i j}^{\mathrm{m}}}\right\} \mathcal{I}\left\{f_{i}\right\}+\mathcal{I}\left\{\mathrm{e}^{2 i \varphi_{i j}^{\mathrm{m}}}\right\} \mathcal{R}\left\{f_{i}\right\}-\mathcal{R}\left\{f_{i}\right\} \\
& =-\mathcal{R}\left\{A_{i j}\right\}+\left(1-2 \sin ^{2} \varphi_{i j}^{\mathrm{m}}\right) \mathcal{R}\left\{f_{i}\right\}-2 \sin \varphi_{i j}^{\mathrm{m}} \cos \varphi_{i j}^{\mathrm{m}} \mathcal{I}\left\{f_{i}\right\}-\mathcal{R}\left\{f_{i}\right\} \\
& =-\mathcal{R}\left\{A_{i j}\right\}+\mathcal{R}\left\{f_{i}\right\}\left(-2 \sin ^{2} \varphi_{i j}^{\mathrm{m}}\right)-\mathcal{I}\left[f_{i}\right] 2 \sin \varphi_{i j}^{\mathrm{m}} \cos \varphi_{i j}^{\mathrm{m}}
\end{aligned}
$$

Equation (A13) now reads

$$
\begin{aligned}
\Delta \varphi_{i j}= & -2 h_{1100, i j}-4 \mathcal{R}\left\{A_{i j}\right\}-8 \sin ^{2} \varphi_{i j}^{\mathrm{m}} \mathcal{R}\left\{f_{i}\right\} \\
& -8 \sin \varphi_{i j}^{\mathrm{m}} \cos \varphi_{i j}^{\mathrm{m}} \mathcal{I}\left\{f_{i}\right\}+O\left(f^{2}\right) \\
= & \bar{h}_{i j}-8 \sin ^{2} \varphi_{i j}^{\mathrm{m}} \mathcal{R}\left\{f_{i}\right\} \\
& -8 \sin \varphi_{i j}^{\mathrm{m}} \cos \varphi_{i j}^{\mathrm{m}} \mathcal{I}\left\{f_{i}\right\}+O\left(f^{2}\right) .
\end{aligned}
$$

We simplified the equation with the definition

$\bar{h}_{i j} \equiv-2 h_{1100, i j}-4 \mathcal{R}\left\{A_{i j}\right\}=\sum_{w \in I} \beta_{w}^{\mathrm{m}} \delta K_{1, w} \sin ^{2} \varphi_{w j}^{\mathrm{m}}$

$\bar{h}_{i j}$ only depends on quadrupole errors inside the range $[i, j]$ and is therefore a local term. The RDTs $f_{i}$ in Eq. (4), on the other hand, contain global contributions.

\section{APPENDIX B: ELIMINATING GLOBAL CONTRIBUTIONS}

The goal of this section is to eliminate global contributions to Eq. (4). This can be achieved by a careful resummation of phase advances between four BPMs.

The global term $\mathcal{R}\left\{f_{i}\right\}$ can be eliminated by taking a third BPM $k$ and divide by the respective factor:

$$
\begin{aligned}
\frac{\Delta \varphi_{i j}}{\sin ^{2} \varphi_{i j}^{\mathrm{m}}}-\frac{\Delta \varphi_{i k}}{\sin ^{2} \varphi_{i k}^{\mathrm{m}}}= & \frac{\bar{h}_{i j}}{\sin ^{2} \varphi_{i j}^{\mathrm{m}}}-\frac{\bar{h}_{i k}}{\sin ^{2} \varphi_{i k}^{\mathrm{m}}} \\
& -8\left(\cot \varphi_{i j}^{\mathrm{m}}-\cot \varphi_{i k}^{\mathrm{m}}\right) \mathcal{I}\left\{f_{i}\right\} .
\end{aligned}
$$

Proceeding similarly with the factor in front of $\mathcal{I}\left\{f_{i}\right\}$,

$$
\frac{\frac{\Delta \varphi_{i j}}{\sin ^{2} \varphi_{i j}^{\mathrm{m}}}-\frac{\Delta \varphi_{i k}}{\sin ^{2} \varphi_{i k}^{\mathrm{m}}}}{\cot \varphi_{i j}^{\mathrm{m}}-\cot \varphi_{i k}^{\mathrm{m}}}=\frac{\frac{\bar{h}_{i j}}{\sin ^{2} \varphi_{i j}^{\mathrm{m}}}-\frac{\bar{h}_{i k}}{\sin ^{2} \varphi_{i k}^{\mathrm{m}}}}{\cot \varphi_{i j}^{\mathrm{m}}-\cot \varphi_{i k}^{\mathrm{m}}}-8 \mathcal{I}\left\{f_{i}\right\} .
$$

We can simplify the lhs to

$$
\begin{aligned}
\frac{\frac{\Delta \varphi_{i j}}{\sin ^{2} \varphi_{i j}^{\mathrm{m}}}-\frac{\Delta \varphi_{i k}}{\sin ^{2} \varphi_{i k}^{\mathrm{m}}}}{\cot \varphi_{i j}^{\mathrm{m}}-\cot \varphi_{i k}^{\mathrm{m}}} & =\frac{\Delta \varphi_{i j}}{\sin ^{2} \varphi_{i j}^{\mathrm{m}}\left(\cot \varphi_{i j}^{\mathrm{m}}-\cot \varphi_{i k}^{\mathrm{m}}\right)}-\frac{\Delta \varphi_{i k}}{\sin ^{2} \varphi_{i k}^{\mathrm{m}}\left(\cot \varphi_{i j}^{\mathrm{m}}-\cot \varphi_{i k}^{\mathrm{m}}\right)} \\
& =\frac{\Delta \varphi_{i j} \sin \varphi_{i j}^{\mathrm{m}} \sin \varphi_{i k}^{\mathrm{m}}}{\sin ^{2} \varphi_{i j}^{\mathrm{m}}\left(\cos \varphi_{i j}^{\mathrm{m}} \sin \varphi_{i k}^{\mathrm{m}}-\cos \varphi_{i k}^{\mathrm{m}} \sin \varphi_{i j}^{\mathrm{m}}\right)}-\frac{\Delta \varphi_{i k} \sin \varphi_{i j}^{\mathrm{m}} \sin \varphi_{i k}^{\mathrm{m}}}{\sin ^{2} \varphi_{i k}^{\mathrm{m}}\left(\cos \varphi_{i j}^{\mathrm{m}} \sin \varphi_{i k}^{\mathrm{m}}-\cos \varphi_{i k}^{\mathrm{m}} \sin \varphi_{i j}^{\mathrm{m}}\right)} \\
& =\frac{\Delta \varphi_{i j}\left(\sin \varphi_{i j}^{\mathrm{m}} \cos \varphi_{j k}^{\mathrm{m}}+\cos \varphi_{i j}^{\mathrm{m}} \sin \varphi_{j k}^{\mathrm{m}}\right)}{\sin \varphi_{i j}^{\mathrm{m}} \sin \varphi_{j k}^{\mathrm{m}}}-\frac{\Delta \varphi_{i k}\left(\sin \varphi_{i k}^{\mathrm{m}} \cos \varphi_{j k}^{\mathrm{m}}-\cos \varphi_{i k}^{\mathrm{m}} \sin \varphi_{j k}^{\mathrm{m}}\right)}{\sin \varphi_{i k}^{\mathrm{m}} \sin \varphi_{j k}^{\mathrm{m}}} \\
& =\Delta \varphi_{i j}\left(\cot \varphi_{i j}^{\mathrm{m}}+\cot \varphi_{j k}^{\mathrm{m}}\right)-\Delta \varphi_{j k}\left(\cot \varphi_{j k}^{\mathrm{m}}-\cot \varphi_{i k}^{\mathrm{m}}\right) .
\end{aligned}
$$

The rhs of Eq. (B2) can be simplified analogously and we can rewrite it to

$$
\begin{aligned}
& \Delta \varphi_{i j}\left(\cot \varphi_{i j}^{\mathrm{m}}+\cot \varphi_{j k}^{\mathrm{m}}\right)-\Delta \varphi_{j k}\left(\cot \varphi_{j k}^{\mathrm{m}}-\cot \varphi_{i k}^{\mathrm{m}}\right) \\
& \quad=\bar{h}_{i j}\left(\cot \varphi_{i j}^{\mathrm{m}}+\cot \varphi_{j k}^{\mathrm{m}}\right)-\bar{h}_{j k}\left(\cot \varphi_{j k}^{\mathrm{m}}-\cot \varphi_{i k}^{\mathrm{m}}\right)-\mathcal{I}\left\{f_{i}\right\}
\end{aligned}
$$


To finally eliminate $\mathcal{I}\left\{f_{i}\right\}$ we take a fourth BPM, $l$, and subtract

$$
\Delta \varphi_{i j}\left(\cot \varphi_{i j}^{\mathrm{m}}+\cot \varphi_{j l}^{\mathrm{m}}\right)-\Delta \varphi_{j l}\left(\cot \varphi_{j l}^{\mathrm{m}}-\cot \varphi_{i l}^{\mathrm{m}}\right)
$$

from Eq. (B4) and end up with

$$
\begin{aligned}
\cot \varphi_{j l}^{\mathrm{m}} & \left(\Delta \varphi_{i l}-\Delta \varphi_{i j}\right)+\cot \varphi_{j k}^{\mathrm{m}}\left(\Delta \varphi_{i j}-\Delta \varphi_{i k}\right) \\
& -\cot \varphi_{k i}^{\mathrm{m}} \Delta \varphi_{i k}+\cot \varphi_{l i}^{\mathrm{m}} \Delta \varphi_{i l} \\
= & \cot \varphi_{j l}^{\mathrm{m}}\left(\bar{h}_{i l}-\bar{h}_{i j}\right)+\cot \varphi_{j k}^{\mathrm{m}}\left(\bar{h}_{i j}-\bar{h}_{i k}\right) \\
& -\cot \varphi_{k i}^{\mathrm{m}} \Delta \varphi_{i k}+\cot \varphi_{l i}^{\mathrm{m}} \bar{h}_{i l}
\end{aligned}
$$

Figure 2 illustrates the collection of four BPMs used to construct Eq. (B5). The left-hand side of Eq. (B5) can be further simplified to

$$
\begin{aligned}
\cot \varphi_{j l}^{\mathrm{m}} & \left(\Delta \varphi_{i l}-\Delta \varphi_{i j}\right)+\cot \varphi_{j k}^{\mathrm{m}}\left(\Delta \varphi_{i j}-\Delta \varphi_{i k}\right) \\
& -\cot \varphi_{k i}^{\mathrm{m}} \Delta \varphi_{i k}+\cot \varphi_{l i}^{\mathrm{m}} \Delta \varphi_{i l} \\
= & \cot \varphi_{j l}^{\mathrm{m}} \Delta \varphi_{j l}-\cot \varphi_{j k}^{\mathrm{m}} \Delta \varphi_{j k}+\cot \varphi_{i k}^{\mathrm{m}} \Delta \varphi_{i k} \\
& -\cot \varphi_{i l}^{\mathrm{m}} \Delta \varphi_{i l} .
\end{aligned}
$$

Now we can rewrite Eq. (B5) as

$$
\Phi_{i j k l}^{\text {meas }}=\Phi_{i j k l}^{\text {model }}
$$

by defining

$$
\begin{aligned}
\Phi_{i j k l}^{\mathrm{meas}} \equiv & \cot \varphi_{j l}^{\mathrm{m}} \Delta \varphi_{j l}-\cot \varphi_{j k}^{\mathrm{m}} \Delta \varphi_{j k} \\
& +\cot \varphi_{i k}^{\mathrm{m}} \Delta \varphi_{i k}-\cot \varphi_{i l}^{\mathrm{m}} \Delta \varphi_{i l}
\end{aligned}
$$

and

$$
\begin{aligned}
\Phi_{i j k l}^{\text {model }} \equiv & \cot \varphi_{j l}^{\mathrm{m}}\left(\bar{h}_{i l}-\bar{h}_{i j}\right)-\cot \varphi_{j k}^{\mathrm{m}}\left(\bar{h}_{i j}-\bar{h}_{i k}\right) \\
& +\cot \varphi_{i k}^{\mathrm{m}} \bar{h}_{i k}-\cot \varphi_{i l}^{\mathrm{m}} \bar{h}_{i l} .
\end{aligned}
$$

Those terms are truly local to the region in between the four BPMs.

\section{APPENDIX C: SECOND ORDER DERIVATIONS}

\section{Second order RDTs}

This section briefly summarizes the calculation of second order resonance driving terms (Appendix A of [25]) and uses the method to calculate the second order contributions from quadrupolar field errors. We use the following conventions:

$$
\begin{aligned}
A^{\ddagger} & \equiv A-\langle A\rangle_{\varphi} \\
A\left(s_{a}\right) & =A_{a},
\end{aligned}
$$

where $A$ is a Hamiltonian term of arbitrary order. The general first order Hamiltonian is given by

$$
\begin{aligned}
H_{w, a}^{(1)}= & \sum_{j_{1} k_{1} l_{1} m_{1}} h_{w, j_{1} k_{1} l_{1} m_{1}} \mathrm{e}^{i\left[\left(j_{1}-k_{1}\right) \varphi_{x, w a}+\left(l_{1}-m_{1}\right) \varphi_{y, w a}\right]} \\
& \times\left(h_{x}^{+}\right)^{j_{1}}\left(h_{x}^{-}\right)^{k_{1}}\left(h_{y}^{+}\right)^{l_{1}}\left(h_{y}^{-}\right)^{m_{1}} .
\end{aligned}
$$

We only consider errors in normal quadrupole components, no perturbation in coupling or higher order multipole terms. Therefore $H^{(1)}$ only contains the following terms:

$$
\begin{gathered}
H_{w, a}^{(1)}=h_{w, 1100} h_{x}^{+} h_{x}^{-}+h_{w, 2000} \mathrm{e}^{2 i \varphi_{w a}}\left(h_{x}^{+}\right)^{2} \\
+h_{w, 0200} \mathrm{e}^{-2 i \varphi_{w a}}\left(h_{x}^{-}\right)^{2} \\
\left\langle H_{w, a}^{(1)}\right\rangle_{\varphi}=h_{w, 1100} h_{x}^{+} h_{x}^{-} \\
H_{w, a}^{(1) \ddagger}=h_{w, 2000} \mathrm{e}^{2 i \varphi_{w a}}\left(h_{x}^{+}\right)^{2}+h_{w, 0200} \mathrm{e}^{-2 i \varphi_{w a}}\left(h_{x}^{-}\right)^{2} .
\end{gathered}
$$

The formula for second order RDTs $F^{(2)}$ from the first and second order Hamiltonian terms $H^{(1)}$ and $H^{(2)}$ is

$$
\begin{aligned}
F_{a}^{(2)}= & \left(H_{a}^{(2) \ddagger}+\frac{1}{2}\left[\left\langle H_{a}^{(1)}\right\rangle_{\varphi}, \frac{1+R}{1-R} H_{a}^{(1) \ddagger}\right]\right. \\
& \left.+\frac{1}{2}\left[H_{a}^{(1) \ddagger}, \frac{H_{a}^{(1) \ddagger}}{1-R}\right]\right) /(1-R) .
\end{aligned}
$$

$F_{a}^{(2)}$ will have, analogously to $F_{a}^{(1)}$, the form

$$
F_{a}^{(2)}=\sum_{j k l m} f_{j k l m}^{(2)}\left(\zeta_{x}^{+}\right)^{j}\left(\zeta_{x}^{-}\right)^{k}\left(\zeta_{y}^{+}\right)^{l}\left(\zeta_{y}^{-}\right)^{m} .
$$

The second order Hamiltonian can be calculated by

$$
H_{a}^{(2)}=\frac{1}{2} \sum_{w}^{W} \sum_{u}^{W}\left[H_{u, a}, H_{w, a}\right]
$$

For the indices $(j k l m)=(2000)$ we get

$$
\begin{aligned}
H_{2000, a}^{(2)}= & \sum_{w}^{W} \sum_{u}^{W}\left\{\left[h_{u, 1100} h_{x}^{+} h_{x}^{-}, h_{w, 2000}\left(h_{x}^{+}\right)^{2}\right]\right. \\
& \left.+\left[h_{u, 2000}\left(h_{x}^{+}\right)^{2}, h_{w, 1100} h_{x}^{+} h_{x}^{-}\right]\right\} \\
= & i h_{2000, a}^{(2)}\left(h_{x}^{+}\right)^{2} \\
\text { with } \quad h_{2000, a}^{(2)}= & \sum_{w}^{W} \sum_{u}^{W}\left\{2 h_{w, 2000} h_{u, 1100} \mathrm{e}^{2 \varphi_{w a}}\right. \\
& \left.-2 h_{w, 1100} h_{u, 2000} \mathrm{e}^{2 \varphi_{u a}}\right\} .
\end{aligned}
$$

In the first row we used $\left[h^{+}, h^{+}\right]=0$ and wrote only those brackets that are nonzero. $h_{0200, a}^{(2)}$ gives a similar result with $h_{w, 2000}$ replaced by $h_{w, 0200}$. Since $\left\langle H_{2000, a}^{(2)}\right\rangle_{\varphi}=0$ we get

$$
H_{2000, a}^{(2) \ddagger}=H_{2000, a}^{(a)} .
$$


The calculation of the bracket in Eq. (C6) yields similar terms when we expand the Poisson brackets and therefore we will not repeat all the steps in detail. The first bracket reads

$$
\begin{aligned}
& \frac{1}{2} \sum_{u, w}^{W}\left[h_{w, 1100} h_{x}^{+} h_{x}^{-}, \frac{1+\mathrm{e}^{4 \pi Q_{x}}}{1-\mathrm{e}^{4 \pi Q_{x}}} h_{u, 2000} \mathrm{e}^{2 \varphi_{u a}}\left(h_{x}^{+}\right)^{2}\right] \\
& =\frac{1}{2} \sum_{u, w}^{W} 2 h_{w, 1100} h_{u, 2000} \cot \left(4 \pi Q_{x}\right) \mathrm{e}^{2 \varphi_{u a}}\left(h_{x}^{+}\right)^{2} \\
& =: h_{2000}^{(1,1)}\left(h_{x}^{+}\right)^{2}
\end{aligned}
$$

and the second one,

$$
\begin{gathered}
{\left[h_{w, 2000} \mathrm{e}^{2 i \varphi_{w a}}\left(h^{+}\right)^{2}+h_{w, 0200} \mathrm{e}^{-2 i \varphi_{w a}}\left(h^{+}\right)^{2},\right.} \\
\left.\frac{h_{w, 2000} \mathrm{e}^{2 i \varphi_{u a}}+h_{w, 0200} \mathrm{e}^{-2 i \varphi_{u a}}}{1-R}\left(h^{+}\right)^{2}\right],
\end{gathered}
$$

reduces to

$$
\begin{aligned}
& \frac{1}{1-e^{4 i \pi Q}}\left(8 i h_{w, 2000} h_{u, 0200} e^{2 i \varphi_{u w}}-8 i h_{w, 0200} h_{u, 2000} e^{-2 i \varphi_{u w}}\right) \\
& \times h_{x}^{+} h_{x}^{-}=: i h_{1100}^{(1,1)} h_{x}^{+} h_{x}^{-} .
\end{aligned}
$$

Up to second order, the RDT can be calculated using

$$
\begin{aligned}
f_{2000} & =f_{2000}^{(1)}+f_{2000}^{(2)} \\
& =f_{2000}^{(1)}+\frac{1}{1-\mathrm{e}^{4 i \pi Q_{x}}}\left\{i h_{2000}^{(2)}+i h_{2000}^{(1,1)}\right\},
\end{aligned}
$$

where $f_{2000}^{(1)}$ is Eq. (A1) and the terms $h_{w, 1100}$ and $h_{w, 2000}$ that appear in the second order expansion are, according to Eq. (A2),

$$
\begin{aligned}
& h_{w, 1100}=\frac{-K_{1, w} \beta_{w}}{4} \\
& h_{w, 2000}=\frac{-K_{1, w} \beta_{w}}{8} .
\end{aligned}
$$

In total we obtain

$$
\begin{aligned}
f_{2000, i}= & \frac{1}{8\left(1-\mathrm{e}^{4 i \pi Q_{x}}\right)} \sum_{w}^{W} K_{1, w} \beta_{w}\left\{\mathrm{e}^{2 \varphi_{w i}}\right. \\
& +\frac{1}{4} \sum_{u}^{w-1} K_{1, u} \beta_{u}\left(e^{2 \varphi_{w i}}-\mathrm{e}^{2 \varphi_{u i}}\right) \\
& \left.+\frac{1}{4} \sum_{u}^{W} \cot \left(4 \pi Q_{x}\right) K_{1, u} \beta_{u}\left(-\mathrm{e}^{2 \varphi_{u i}}\right)\right\} \\
& +O\left(K^{3}\right) .
\end{aligned}
$$

\section{Second order tune shift}

The tune shift comes from the phase-independent Hamiltonian in normal forms

$$
\mathbf{H}_{\text {norm }}=\mathbf{H}_{\text {norm }}^{(1)}+\mathbf{H}_{\text {norm }}^{(2)} .
$$

The first order part of the phase independent Hamiltonian is just the phase average of the first order Hamiltonian in Courant-Snyder coordinates

$$
\mathbf{H}_{\text {norm }}^{(1)}=\left\langle H^{(1)}\right\rangle_{\varphi}
$$

whereas the second order term is calculated by

$$
\mathbf{H}_{\mathrm{norm}}^{(2)}=\left\langle H^{(2)}\right\rangle_{\varphi}+\frac{1}{2}\left\langle\left[H^{(1) \ddagger}, \frac{H^{(1) \ddagger}}{1-R}\right]\right\rangle_{\varphi} .
$$

Again, the Poisson bracket yields $h_{1100}^{(1,1)}$ of Eq. (C13)

$$
h_{1100}^{(1,1)}=\sum_{w}^{W} \sum_{u}^{W} \frac{K_{1, u} K_{1, w} \beta_{u} \beta_{w}}{8\left(1-e^{4 i \pi Q}\right)} \sin \left(2 \varphi_{u w}\right),
$$

and $\left\langle H^{(2)}\right\rangle_{\varphi}$ is the sum over all phase independent terms, i.e., $j=k$ :

$$
\begin{aligned}
\sum_{w}^{W} & \sum_{u}^{W}\left\{4 h_{w, 2000} h_{u, 0200} \mathrm{e}^{2 i \varphi_{w i}-2 i \varphi_{u i}}\right. \\
& \left.-4 h_{w, 0200} h_{u, 2000} \mathrm{e}^{2 i \varphi_{u i}-2 i \varphi_{w i}}\right\} \\
= & \sum_{w}^{W} \sum_{u}^{W} \frac{K_{1, u} K_{1, w} \beta_{u} \beta_{w}}{16} i \sin \left(2 \varphi_{w u}\right)=: i h_{1100}^{(2)} .
\end{aligned}
$$

The contribution to the phase advance is the accumulated phase shift between elements $i$ and $j$ :

$$
\begin{aligned}
& h_{1100, i j}^{(2)}+h_{1100, i j}^{(1,1)} \\
& =\operatorname{sgn}(j-i) \sum_{w \in I} \sum_{u}^{W} \frac{K_{1, u} K_{1, w} \beta_{u} \beta_{w}}{16} \\
& \quad \times \sin \left(2 \varphi_{w u}\right)\left(1+\frac{1}{1-e^{4 i \pi Q}}\right) .
\end{aligned}
$$

\section{Phase beating - second order}

Combining Eqs. (A6) and (A8) while keeping all terms up to second order, we obtain the following expression for the second order phases advance beating Eq. (4): 


$$
\begin{aligned}
\Delta \varphi_{i j}= & -2 h_{1100, i j}^{(1)}-2 h_{1100, i j}^{(2)}-2 h_{1100, i j}^{(1,1)} \\
& +4 \mathcal{R}\left\{f_{j}^{(1)}-f_{i}^{(1)}\right\}+4 \mathcal{R}\left\{f_{j}^{(2)}-f_{i}^{(2)}\right\} \\
& +16\left(\mathcal{R}\left\{f_{j}^{(1)}\right\} \mathcal{I}\left\{f_{j}^{(1)}\right\}-\mathcal{R}\left\{f_{i}^{(1)}\right\} \mathcal{I}\left\{f_{i}^{(1)}\right\}\right) \\
& +O\left(K^{3}\right) .
\end{aligned}
$$

The superscripts (1) and (2) denote first and second order. The second order terms contain global error contributions that cannot be eliminated by a new reshuffling and resummation because of the nested sums in Eqs. (C17) and $(\mathrm{C} 23)$.

[1] R. Tomás, M. Aiba, A. Franchi, and U. Iriso, Review of linear optics measurement and correction for charged particle accelerators, Phys. Rev. Accel. Beams 20, 054801 (2017).

[2] R. Tomás, T. Bach, R. Calaga, A. Langner, Y. I. Levinsen, E. H. MacLean, T. H. Persson, P. K. Skowronski, M. Strzelczyk, G. Vanbavinckhove, and R. Miyamoto, Record low $\beta$ beating in the LHC, Phys. Rev. ST Accel. Beams 15, 091001 (2012).

[3] T. Persson, F. Carlier, J.C. De Portugal, A. G. T. Valdivieso, A. Langner, E. H. Maclean, L. Malina, P. Skowronski, B. Salvant, R. Tomás, and A. C. Bonilla, LHC optics commissioning: A journey towards $1 \%$ optics control, Phys. Rev. Accel. Beams 20, 061002 (2017).

[4] M. Aiba, M. Böge, J. Chrin, N. Milas, T. Schilcher, and A. Streun, Comparison of linear optics measurement and correction methods at the Swiss Light Source, Phys. Rev. ST Accel. Beams 16, 012802 (2013).

[5] D. Sagan, R. Meller, R. Littauer, and D. Rubin, Betatron phase and coupling measurements at the Cornell electron/ positron storage ring, Phys. Rev. ST Accel. Beams 3, 092801 (2000).

[6] J. Borer, A. Hofmann, J. Koutchouk, T. Risselada, and B. Zotter, Measurements of betatron phase advance and beta function in the ISR, IEEE Trans. Nucl. Sci. 30, 2406 (1983).

[7] A. Langner, G. Benedetti, M. Carlà, J. M. C. de Portugal, U. Iriso, Z. Martí, and R. Tomás, Optics measurement using the N-BPM method for the ALBA synchrotron, in Proceedings of the 6th International Particle Accelerator Conference (IPAC 2015), Richmond, Virginia (CERN, Geneva, Switzerland, 2015), MOPJE057.

[8] J. C. de Portugal, R. Tomás, and M. Hofer, New local optics measurements and correction techniques for the LHC and its luminosity upgrade, Phys. Rev. Accel. Beams 23, 041001 (2020).

[9] R. Tomás, M. Bai, R. Calaga, W. Fischer, A. Franchi, and G. Rumolo, Measurement of global and local resonance terms, Phys. Rev. ST Accel. Beams 8, 024001 (2005).

[10] A. Franchi, R. Tomás, and F. Schmidt, Magnet strength measurement in circular accelerators from beam position monitor data, Phys. Rev. ST Accel. Beams 10, 074001 (2007).

[11] A. Franchi and R. Tomás, Computation of the coupling resonance driving term $\mathrm{f} 1001$ and the coupling coefficient
C from turn-by-turn single-BPM data, CERN Technical Report No. CERN-BE-Note-2010-016, 2010.

[12] R. Miyamoto, Measurement of coupling resonance driving terms in the LHC with AC dipoles, Technical Report, 2010.

[13] P. Castro, Luminosity and beta function measurement at the electron-positron collider ring LEP, Ph.d thesis, CERN, Geneva, 1996 [Report No. CERN-BE-Note-2010-016].

[14] R. Calaga, R. Tomás, and F. Zimmermann, BPM calibration independent LHC optics correction, 2007 IEEE Particle Accelerator Conference (PAC) (2007), pp. 3693-3695, https://accelconf.web.cern.ch/p07/ PAPERS/THPAS091.PDF.

[15] W. Guo, S. L. Kramer, F. Willeke, X. Yang, and L. Yu, A lattice correction approach through betatron phase advance, in Proceedings of the 7th International Particle Accelerator Conference (IPAC 2016), Busan, Korea, 2016 (CERN, JACoW, 2016), MOOCB02, https://doi.org/ 10.18429/JACoW-IPAC2016-MOOCB02.

[16] J. Laskar, C. Froeschle, and A. Celletti, The measure of chaos by the numerical analysis of the fundamental frequencies. Application to the standard mapping, Physica (Amsterdam) 56D, 253 (1992).

[17] R. Miyamoto, S. E. Kopp, A. Jansson, and M. J. Syphers, Parametrization of the driven betatron oscillation, Phys. Rev. ST Accel. Beams 11, 084002 (2008).

[18] T. Zhang, X. Huang, and T. Maxwell, Linear optics correction for linacs and free electron lasers, Phys. Rev. Accel. Beams 21, 092801 (2018).

[19] R. Bartolini and F. Schmidt, Normal form via tracking or beam data, Part. Accel. 59, 93 (1997).

[20] S. Fartoukh, Achromatic telescopic squeezing scheme and application to the LHC and its luminosity upgrade, Phys. Rev. ST Accel. Beams 16, 111002 (2013).

[21] P. Hagen, M. Giovannozzi, J. P. Koutchouk, T. Risselada, S. Sanfilippo, E. Todesco, and E. Wildner, WISE: An adaptive simulation of the LHC optics, Conf. Proc. C060626, 2248 (2006).

[22] P. Hagen, M. Giovannozzi, J. P. Koutchouk, T. Risselada, F. Schmidt, E. Todesco, and E. Y. Wildner, WISE: A simulation of the LHC optics including magnet geometrical data, Conf. Proc. C0806233, TUPP091 (2008).

[23] R. Calaga and R. Tomás, Statistical analysis of RHIC beam position monitors performance, Phys. Rev. ST Accel. Beams 7, 042801 (2004).

[24] M. Gasior, J. Olexa, and R. J. Steinhagen, A highresolution diode-based orbit measurement systemPrototype results from the LHC, Conf. Proc. C11-0516.4, MOPD24 (2011).

[25] A. Franchi, L. Farvacque, F. Ewald, G. Le Bec, and K. B. Scheidt, First simultaneous measurement of sextupolar and octupolar resonance driving terms in a circular accelerator from turn-by-turn beam position monitor data, Phys. Rev. ST Accel. Beams 17, 074001 (2014).

[26] A. Franchi, Error analysis of linear optics measurements via turn-by-turn beam position data in circular accelerators, arXiv:1603.00281.

[27] A. Bazzani, E. Todesco, G. Turchetti, and G. Servizi, A normal form approach to the theory of nonlinear betatronic motion, CERN Yellow Reports No. CERN-94-02, No. CERN-YELLOW-94-02, 1994. 